\title{
Landform Degradation on Mercury, the Moon, and Mars: Evidence From Crater Depth/Diameter Relationships
}

\author{
Michael C. Malin ${ }^{1}$ and Daniel Dzurisin \\ Division of Geological and Planetary Sciences, California Institute of Technology, Pasadena, California 91125
}

\begin{abstract}
Morphologic classification of craters and quantitative measurements of crater depth as a function of diameter are used to investigate the relative degradational histories of Mercury, the moon, and Mars. Martian craters exhibit considerable depth variation and are generally shallower than their lunar or mercurian counterparts. On Mercury and the moon, visually fresh and degraded craters on smooth plains show no significant depth degradation except that attributed to lava flooding or local inundation by ejecta from large impacts. More heavily cratered regions on both planets display a large range of both visual and depth degradation, suggesting that most landform modification occurred before the final phase of formation of the oldest smooth plains on both planets. Depth/diameter data presented here are discussed as they relate to two early history scenarios. One scenario based on cratering and the ballistic transport of material has been suggested for Mercury, the moon, and Mars by several authors. Owing to discrepancies between this ballistic scenario and observations of crater densities and morphologies, we suggest that landforms on all these bodies also record nonballistic degradation associated with the formation of intercrater plains. Whichever scenario is applied, early, intense, bombardment-associated degradation appears to be a common element in the histories of the terrestrial planets.
\end{abstract}

\section{INTRODUCTION}

Features seen on the surfaces of terrestrial planets are the results of numerous constructive and/or destructive processes. Impact cratering has been an important constructive process on Mercury, the moon, and Mars. On all three planets the abundance of craters makes them convenient features to study as a means of evaluating processes which modify landscapes.

This work investigates degradation, by which we mean the deterioration of landforms. This deterioration may be the result of depositional or erosional processes which are externally controlled (e.g., ballistic or eolian transport), or it may result from internally generated processes which diminish relief (e.g., mare flooding, isostatic readjustment).

Comparisons of crater degradation are made on three terrain units common to Mercury, the moon, and Mars. Two units are distinguished by the relative abundances of superimposed craters: smooth plains (few large craters) and heavily cratered terrain (clusters of large craters). Large areas of moderately cratered surface, which separate clusters of craters within the more heavily cratered portions of the planets, are called intercrater plains [Trask and Guest, 1975].

\section{Crater Morphology and Depth/Diameter Relationships Morphologic Indicators}

Features used by previous investigators to recognize morphologic modification of craters with time include crater rim crispness and the presence or absence of continuous ejecta deposits and secondary impact features [e.g., Pohn and Offield. 1970; Arthur, 1963, 1974; Pike, 1974]. Terraced interior walls, central peaks or rings, and crater floor morphology have been interpreted as products of postimpact mass movements, such as slumping or landsliding from crater walls, or isostatic rebound of crater floors [e.g., Pike, 1974; Head, 1975]. We use the term 'visual degradation' to indicate the deteriora-

\footnotetext{
${ }^{1}$ Now at Planetology and Oceanography Section, Jet Propulsion Laboratory, California Institute of Technology, Pasadena, California 91103 .

Copyright (؟ 1977 by the American Geophysical Union.
}

tion of these morphologic features. 'Combined with quantitative measurements of crater depth (indicating 'depth degradation') as a function of diameter, such qualitative indicators provide a basis for evaluation of the relative degradational histories of Mercury, the moon, and Mars. In practice, however, differences in resolution, lighting, or viewing geometry often complicate the consistent application of these indicators to craters on different planets. Additionally, systematic biases associated with various techniques employed to measure crater depth (e.g., radar range measurements, shadow analysis, photogrammetry) limit the extent to which data sets can be usefully compared.

To minimize the effects of such difficulties, we have, whenever possible, relied upon redundant photographic coverage to provide a range in lighting and viewing geometries, or we have considered only craters for which favorable lighting and viewing geometries are available. Published morphologic indicators for lunar craters have been generalized to facilitate comparison with mercurian and martian craters.

\section{Morphologic Classification of Craters on Mercury}

Mariner 10 television imaging provided coverage of roughly $40 \%$ of the surface of Mercury at resolutions ranging from 1 to $3 \mathrm{~km}$ and a limited number of specially targeted views with resolution as high as $120 \mathrm{~m}$. A total of 449 craters ranging in diameter from 1 to $200 \mathrm{~km}$ have been chosen for study. The latitudinal distribution of these craters is essentially random, ranging from $80^{\circ} \mathrm{N}$ to $90^{\circ} \mathrm{S}$; longitudes are restricted to between $10^{\circ} \mathrm{W}$ and $40^{\circ} \mathrm{W}$ and between $160^{\circ} \mathrm{W}$ and $190^{\circ} \mathrm{W}$, ow-

TABLE 1. Physiographic Distribution of Mercurian Craters

\begin{tabular}{lccc}
\hline & $\begin{array}{c}\text { Fresh } \\
\text { (Class I) }\end{array}$ & $\begin{array}{c}\text { Degraded } \\
\text { (Class 2) }\end{array}$ & Total \\
\hline Smooth plains & 90 & 59 & 149 \\
Intercrater plains & 51 & 112 & 163 \\
Heavily cratered terrain & 37 & 100 & 137 \\
Total & 178 & 271 & 449 \\
\hline
\end{tabular}

Terrain units after Trask and Guest [1975]. 


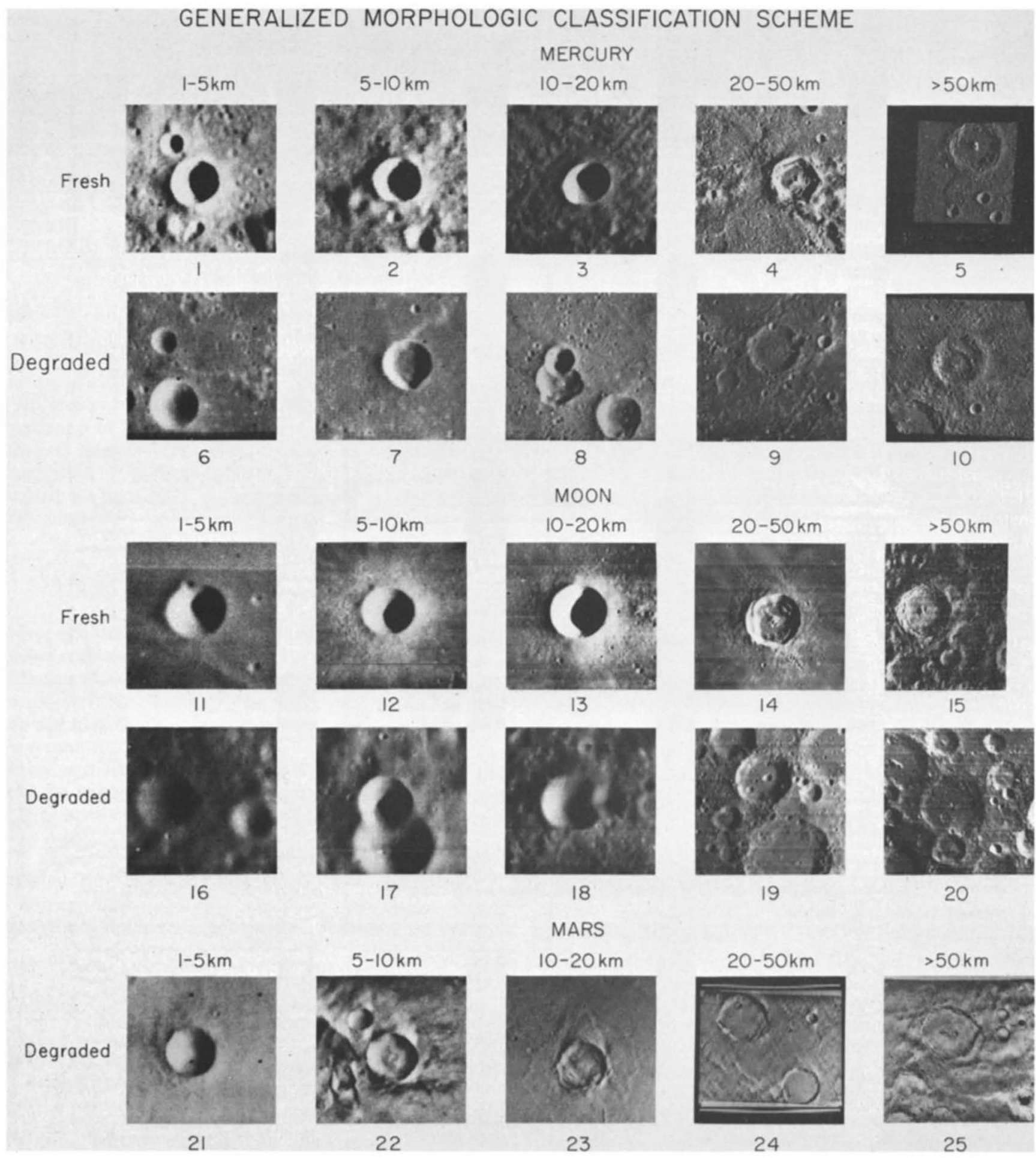

Fig. 1. Generalized two-class morphologic classification scheme used to facilitate comparison of widely differing photographic data sets on Mercury, the moon, and Mars. Only the freshest craters in each size range were grouped as class 1 ; all others were classified as degraded (class 2). Lunar images are from Lunar Orbiter 4 (resolution, $\sim 60 \mathrm{~m}$ ), mercurian images from Mariner 10 (resolution, $120 \mathrm{~m}$ to $3 \mathrm{~km}$ ), and martian images from Mariner 9 (resolution, $100 \mathrm{~m}$ to $1 \mathrm{~km}$ ). The numbers (1-25) beneath the individual images are keyed to Table 2, which contains supporting information.

ing to viewing and lighting constraints. The distribution of craters by physiographic province is shown in Table 1.

Owing to resolution limitations, craters on Mercury are grouped simply as fresh (class 1 ) or degraded (class 2 ) on the basis of a qualitative assessment using the indicators discussed above. In general, craters less than $10 \mathrm{~km}$ in diameter with crisp rims, continuous ejecta, and/or satellitic crater fields and with apparent bowl-shaped morphology were assigned to class 1 ; all others were assigned to class 2 . For craters larger than 10 $\mathrm{km}$ in diameter, morphologically fresh wall terraces and cen- 
TABLE 2. Supporting Data for Figure 1

\begin{tabular}{|c|c|c|c|c|}
\hline $\begin{array}{l}\text { Image on } \\
\text { Figure } 1\end{array}$ & $\begin{array}{c}\text { Picture } \\
\text { Identification }\end{array}$ & $\begin{array}{l}\text { Resolution, } \\
\text { km }\end{array}$ & $\begin{array}{l}\text { Latitude, } \\
\text { deg }\end{array}$ & $\begin{array}{c}\text { Longitude } \\
\text { deg }\end{array}$ \\
\hline \multicolumn{5}{|c|}{ Mariner 10} \\
\hline 1 & 528978 & 0.21 & 17 & 172 \\
\hline 2 & 076 & 0.40 & 37 & 172 \\
\hline 3 & 056 & 0.24 & -2 & 166 \\
\hline 4 & 074 & 0.36 & 30 & 168 \\
\hline 5 & 080 & 0.50 & 59 & 180 \\
\hline 6 & 528991 & 0.34 & 27 & 148 \\
\hline 7 & 070 & 0.32 & 17 & 166 \\
\hline 8 & 071 & 0.34 & 21 & 168 \\
\hline 9 & 092 & 0.60 & 64 & 159 \\
\hline 10 & 27458 & 0.52 & 9 & 22 \\
\hline \multicolumn{5}{|c|}{ Lunar Orbiter 4} \\
\hline 11 & $134 \mathrm{H} 1$ & 0.062 & 46 & -18 \\
\hline 12 & $113 \mathrm{H3}$ & 0.059 & -3 & -8 \\
\hline 13 & $132 \mathrm{H} 3$ & 0.060 & -8 & -30 \\
\hline 14 & $133 \mathrm{H} 3$ & 0.058 & 0 & -28 \\
\hline 15 & $119 \mathrm{H} 2$ & 0.066 & -43 & -11 \\
\hline 16 & $95 \mathrm{H3}$ & 0.065 & -27 & 11 \\
\hline 17 & $95 \mathrm{H3}$ & 0.065 & -27 & 14 \\
\hline 18 & $95 \mathrm{H3}$ & 0.065 & -29 & 12 \\
\hline 19 & $100 \mathrm{H1}$ & 0.065 & -42 & 12 \\
\hline 20 & $70 \mathrm{H} 3$ & 0.076 & -56 & 44 \\
\hline \multicolumn{5}{|c|}{ Mariner 9} \\
\hline 21 & 7003223 & 0.13 & 3 & 304 \\
\hline 22 & 7002663 & 0.10 & -13 & 309 \\
\hline 23 & 7435333 & 0.14 & 15 & 245 \\
\hline 24 & 7435193 & 0.14 & 6 & 249 \\
\hline 25 & 6859198 & 1.24 & 3 & 322 \\
\hline
\end{tabular}

tral peaks or rings were used as additional indicators of crater freshness. Examples of fresh and degraded mercurian craters of various diameters are given in Figure 1 (Table 2).

\section{Morphologic Classification \\ of Craters on the Moon}

Depth/diameter curves for lunar craters in several degradational states have been generated from data presented by Pike [1974] and Arthur [1974]. Pike's study included 208 fresh craters distributed on mare and terra surfaces. Arthur presented nearly 2000 lunar craters divided into five degradational classes, class 1 including pristine craters and class 5 the most degraded craters discernible (Table 3 ). The relationship between the states of degradation defined above for Mercury and those defined by Pike and Arthur for the moon as determined by comparison of similarly sized craters in various classes on both bodies is shown in Figure 1 and Table 4 .

Difficulty was encountered in generalizing the five-class deg-

TABLE 3. Physiographic Distribution of Lunar Craters

\begin{tabular}{lccc}
\hline & $\begin{array}{c}\text { Fresh } \\
\text { (Class 1) }\end{array}$ & $\begin{array}{c}\text { Degraded } \\
\text { (Classes 2-5) }\end{array}$ & Total \\
\hline Mare & \multicolumn{2}{c}{ Arthur $[1974]$} & \\
Terra & 548 & 94 & 642 \\
Total & 509 & 623 & 1132 \\
& 1057 & 717 & 1774 \\
Mare & \multicolumn{2}{c}{ Pike $[1974]$} & \\
Terra & 146 & & 146 \\
Total & 52 & & 52 \\
\hline
\end{tabular}

TABLE 4. Generalized Morphologic Classification Scheme

\begin{tabular}{llll}
\hline & & \multicolumn{2}{c}{ Degradational State } \\
\cline { 3 - 4 } Planet & Source & Fresh & Degraded \\
\hline Mercury & This paper & class 1 & class 2 \\
Moon & $\begin{array}{l}\text { Arthur [1974] } \\
\text { Pike [1974] } \\
\text { This paper }\end{array}$ & class 1 & classes 2-5 \\
Mars & class 1 & class 2 \\
\hline
\end{tabular}

radational scheme to a two-class system. Class 1 lunar craters were easily correlated with class 1 craters on Mercury, and lunar classes 3-5 corresponded morphologically to mercurian class 2 . However, class 2 lunar craters could not be unambiguously assigned to either the fresh or the degraded class. These class 2 craters have been grouped with classes 3-5 as degraded to facilitate direct comparison of the freshest (class 1) craters on Mercury and the moon. Except for degraded lunar mare craters this arrangement does not significantly alter the form of the depth/diameter curves for lunar craters. Separate plots including classes 2-5 and 3-5 are provided (Figure 5) for degraded lunar mare craters.

\section{Morphologic Classification of Craters on Mars}

The extensive history of eolian and volcanic deposition and erosion inferred from crater forms on Mars further complicates application of the simple mercurian classification scheme. Although some martian craters with central peaks, satellitic crater fields, and/or continuous ejecta deposits exist, there is other evidence that all 178 martian craters studied have been at least superficially mantled at some time in their history, and hence all are placed in class 2 . Consistent with the generalized scheme developed above, apparent degradation of these craters ranges from slight to nearly complete.

\section{Depth/Diameter Relationships}

Mercury. Shadow analysis techniques applied to high-resolution near-terminator photography from Mariner 10 provide the basis for quantitative studies of crater depth as a function of diameter for both fresh and degraded mercurian craters. Results for 178 fresh and 271 degraded craters (Table 5 ) are shown in Figure 2, and the data are grouped by physiographic province in Figure 3.

Pike [1974] emphasizes that shadow analysis techniques can yield depths for small lunar craters which are as much as $20 \%$ too shallow, although the average is about $5 \%$. Such discrepancies presumably result from resolution limitations and from encroachment of the shadow cast by one crater wall onto the inward sloping face of the opposite wall. Such effects have been minimized in the Mercury data by (1) eliminating craters less than 10 picture elements in diameter and (2) measuring only craters over a wide range in sun elevation angles whose shadows approximately bisect their diameters.

The moon. Previously published depth/diameter curves for both fresh and degraded lunar craters are shown in Figure 4. Pike [1974] studied fresh lunar craters by stereo photogrammetry applied to Apollo metric camera frames. The data presented by Arthur [1974] were determined by using shadow analysis techniques to measure depths for both fresh and degraded lunar craters imaged by Lunar Orbiter 4 . Although Pike [1974] noted that these shadow measurements may be underestimates of the true depths of fresh craters (a point 
TABLE 5a. Mercury Class 1

TABLE 5a. (continued)

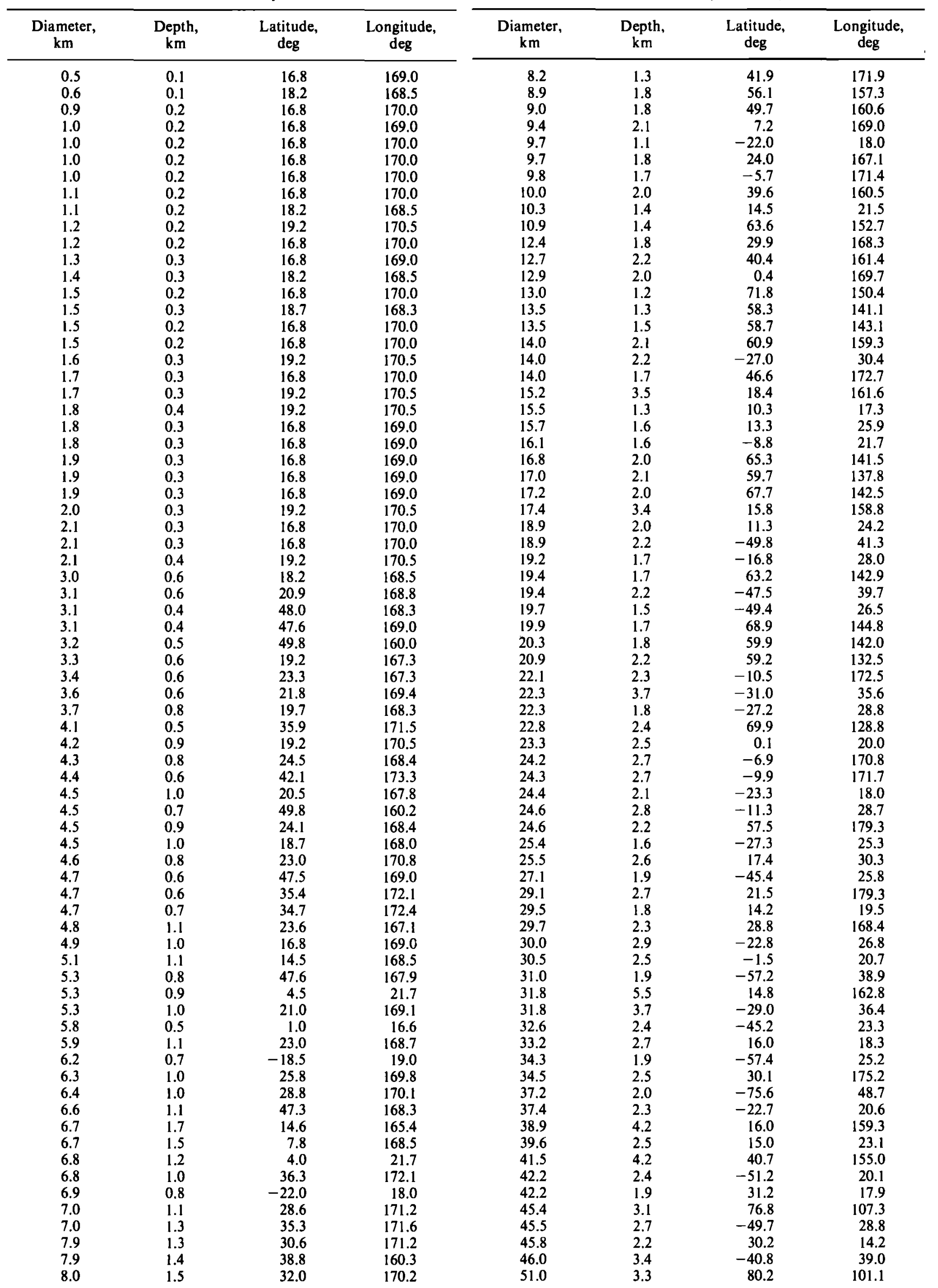


TABLE 5a. (continued)

\begin{tabular}{crrr}
\hline $\begin{array}{c}\text { Diameter, } \\
\text { km }\end{array}$ & $\begin{array}{c}\text { Depth, } \\
\text { km }\end{array}$ & $\begin{array}{c}\text { Latitude, } \\
\text { deg }\end{array}$ & $\begin{array}{c}\text { Longitude, } \\
\text { deg }\end{array}$ \\
\hline 51.3 & 3.1 & 32.7 & 176.5 \\
51.4 & 1.7 & 31.9 & 18.5 \\
52.0 & 3.8 & 54.9 & 154.6 \\
55.1 & 1.8 & 1.7 & 15.8 \\
55.2 & 2.7 & 71.0 & 176.6 \\
55.4 & 3.0 & -63.9 & 35.0 \\
56.9 & 2.5 & 23.6 & 179.0 \\
57.7 & 2.5 & 65.2 & 132.6 \\
58.3 & 3.0 & 72.5 & 111.2 \\
58.6 & 1.7 & 33.0 & 15.3 \\
62.6 & 2.4 & 51.3 & 176.8 \\
63.9 & 2.4 & -13.7 & 14.2 \\
65.8 & 3.5 & 40.6 & 178.5 \\
69.5 & 2.3 & 6.4 & 14.8 \\
71.4 & 6.3 & 32.6 & 164.5 \\
71.4 & 3.7 & 28.9 & 174.6 \\
72.9 & 3.8 & 62.0 & 136.7 \\
73.7 & 5.2 & -10.9 & 31.5 \\
75.1 & 3.6 & -65.3 & 31.4 \\
80.7 & 3.0 & -40.4 & 26.5 \\
89.1 & 4.0 & -62.0 & 22.8 \\
89.6 & 2.5 & -10.1 & 12.8 \\
93.9 & 2.7 & 59.9 & 175.3 \\
100.4 & 2.0 & 3.3 & 14.6 \\
102.2 & 3.6 & 12.5 & 24.4 \\
111.0 & 2.2 & -15.8 & 17.4 \\
116.0 & 2.4 & 35.5 & 18.1 \\
118.0 & 2.8 & -19.6 & 19.9 \\
118.2 & 3.7 & -38.3 & 32.4 \\
132.1 & 2.2 & 66.2 & 163.8 \\
133.8 & 2.8 & 19.0 & 24.8 \\
160.2 & 3.7 & -79.9 & 36.8 \\
\hline & & &
\end{tabular}

demonstrated by least squares fits to the data), in practice, the two populations for fresh craters in Figure 4 are barely distinguishable. In Figures 5 and 6, craters presented by Pike [1974] and Arthur [1974] are grouped by physiographic province.

Mars. Data from three distinct sources (Table 6) have been combined to produce the depth/diameter curve for martian craters shown in Figure 7. Comparison of depth measurements for similarly sized craters obtained by different techniques suggests that systematic differences between techniques are relatively minor. At small diameters $(1<D<10 \mathrm{~km})$, depths were derived from shadow analysis of 106 craters appearing in near-terminator, narrow-angle Mariner 9 images. The average sun elevation angle for frames studied was about $5^{\circ}$. To the extent that photometric shading may have been mistaken for true shadows, these measurements represent upper limits for true crater depths. The accuracy of the depth determinations is estimated to be roughly $20 \%$. To avoid the problems noted by Pike [1974], craters less than 10 picture elements in diameter were eliminated, as were those with potentially serious shadow projection effects.

Photoclinometric analysis of Mariner 4 images provides depth/diameter information for 41 craters ranging from 5 to $40 \mathrm{~km}$ in diameter [Leighton, 1966]. Depths are reportedly accurate to roughly $100 \mathrm{~m}$. However, it must be cautioned that photoclinometric analyses of more recent higher-resolution, better quality images of Mars have been largely unsuccessful, since albedo variations cannot be distinguished from changes in slope. J. Veverka (personal communication, 1975) suggests that Mariner 9 ultraviolet spectrometer data indicate a statistically deeper population of $12-$ to $50-\mathrm{km}$ craters than is presented here. However, since a number of craters lying along
TABLE $5 b$. Mercury Class 2

\begin{tabular}{|c|c|c|c|}
\hline $\begin{array}{c}\text { Diameter, } \\
\mathbf{k m}\end{array}$ & $\begin{array}{c}\text { Depth, } \\
\text { km }\end{array}$ & $\begin{array}{c}\text { Latitude, } \\
\text { deg }\end{array}$ & $\begin{array}{c}\text { Longitude, } \\
\text { deg }\end{array}$ \\
\hline 2.1 & 0.3 & 18.5 & 168.1 \\
\hline 2.3 & 0.2 & -12.0 & 28.0 \\
\hline 2.3 & 0.3 & -12.0 & 28.0 \\
\hline 2.5 & 0.3 & 18.6 & 168.3 \\
\hline 2.5 & 0.3 & 18.4 & 168.3 \\
\hline 3.3 & 0.5 & 25.8 & 170.7 \\
\hline 3.5 & 0.5 & -11.0 & 28.0 \\
\hline 3.8 & 0.7 & 18.9 & 166.8 \\
\hline 3.8 & 0.7 & 14.7 & 167.9 \\
\hline 4.0 & 0.7 & 24.9 & 167.8 \\
\hline 4.1 & 0.6 & 25.2 & 170.8 \\
\hline 4.1 & 0.2 & -21.0 & 18.0 \\
\hline 4.5 & 0.8 & 19.2 & 165.5 \\
\hline 4.6 & 0.4 & -11.0 & 27.0 \\
\hline 4.7 & 0.8 & -8.7 & 172.3 \\
\hline 4.8 & 0.8 & -9.0 & 172.8 \\
\hline 4.8 & 1.0 & 24.5 & 167.7 \\
\hline 5.1 & 0.5 & -44.0 & 25.0 \\
\hline 5.1 & 0.5 & -44.0 & 25.0 \\
\hline 5.3 & 0.7 & 40.9 & 170.6 \\
\hline 5.3 & 0.4 & 2.0 & 20.0 \\
\hline 5.3 & 1.0 & 26.2 & 169.7 \\
\hline 5.5 & 1.0 & 14.5 & 167.3 \\
\hline 6.0 & 1.1 & 25.7 & 167.5 \\
\hline 6.1 & 1.0 & 29.3 & 168.2 \\
\hline 6.1 & 1.1 & 32.4 & 170.4 \\
\hline 6.2 & 1.2 & 39.4 & 160.5 \\
\hline 6.4 & 0.2 & 1.0 & 17.0 \\
\hline 6.5 & 1.4 & 12.3 & 168.3 \\
\hline 6.7 & 1.3 & 25.9 & 167.1 \\
\hline 6.8 & 0.2 & 2.0 & 19.0 \\
\hline 6.8 & 0.5 & -42.0 & 21.0 \\
\hline 6.8 & 0.5 & -41.0 & 22.0 \\
\hline 6.9 & 1.4 & 39.1 & 160.5 \\
\hline 6.9 & 1.5 & 14.2 & 166.1 \\
\hline 7.2 & 1.2 & -8.4 & 173.3 \\
\hline 7.2 & 1.4 & 13.0 & 167.6 \\
\hline 7.2 & 1.3 & 40.6 & 170.1 \\
\hline 7.9 & 1.3 & -8.8 & 173.0 \\
\hline 8.8 & 1.2 & 18.7 & 165.4 \\
\hline 9.1 & 1.0 & 50.6 & 155.4 \\
\hline 9.1 & 1.8 & 20.4 & 169.4 \\
\hline 9.3 & 1.9 & 39.0 & 161.3 \\
\hline 9.6 & 2.4 & 0.5 & 165.4 \\
\hline 9.9 & 1.9 & -5.7 & 172.2 \\
\hline 9.9 & 1.4 & 53.3 & 152.4 \\
\hline 10.1 & 0.8 & -40.0 & 27.0 \\
\hline 10.2 & 1.5 & 51.7 & 153.0 \\
\hline 10.3 & 0.9 & -1.7 & 15.0 \\
\hline 10.3 & 0.4 & 0.5 & 19.2 \\
\hline 11.2 & 1.7 & 10.0 & 166.9 \\
\hline 11.3 & 1.3 & 59.7 & 141.3 \\
\hline 11.4 & 0.5 & 5.4 & 13.6 \\
\hline 11.6 & 0.7 & 69.9 & 156.3 \\
\hline 11.6 & 0.5 & 1.0 & 19.0 \\
\hline 11.8 & 0.7 & -42.0 & 21.0 \\
\hline 11.8 & 0.9 & -44.0 & 33.0 \\
\hline 12.0 & 1.0 & 2.0 & 20.0 \\
\hline 12.0 & 0.7 & 0.8 & 20.0 \\
\hline 12.2 & 0.3 & 4.1 & 12.9 \\
\hline 12.3 & 0.3 & 69.5 & 155.4 \\
\hline 12.5 & 2.3 & 0.9 & 167.8 \\
\hline 12.6 & 2.8 & 17.9 & 159.8 \\
\hline 13.0 & 3.4 & 15.3 & 157.7 \\
\hline 13.0 & 1.7 & 18.5 & 166.1 \\
\hline 13.3 & 1.5 & 50.7 & 154.9 \\
\hline 13.5 & 0.7 & -44.5 & 24.5 \\
\hline 13.5 & 0.7 & -44.0 & 25.0 \\
\hline 13.8 & 3.0 & 14.5 & 163.4 \\
\hline 14.0 & 1.2 & 21.7 & 168.7 \\
\hline 14.1 & 2.2 & 40.5 & 157.9 \\
\hline 14.1 & 1.4 & 70.5 & 124.7 \\
\hline 14.2 & 1.4 & 52.3 & 154.4 \\
\hline
\end{tabular}


TABLE $5 b$. (continued)

\begin{tabular}{|c|c|c|c|c|c|c|c|}
\hline $\begin{array}{c}\text { Diameter, } \\
\mathrm{km}\end{array}$ & $\begin{array}{c}\text { Depth, } \\
\text { km }\end{array}$ & $\begin{array}{l}\text { Latitude, } \\
\text { deg }\end{array}$ & $\begin{array}{c}\text { Longitude, } \\
\text { deg }\end{array}$ & $\begin{array}{c}\text { Diameter, } \\
\text { km }\end{array}$ & $\begin{array}{l}\text { Depth, } \\
\text { km }\end{array}$ & $\begin{array}{l}\text { Latitude, } \\
\text { deg }\end{array}$ & $\begin{array}{c}\text { Longitude, } \\
\text { deg }\end{array}$ \\
\hline 14.3 & 0.6 & 6.8 & 19.0 & 29.3 & 2.5 & 68.8 & 128.8 \\
\hline 14.3 & 0.3 & 73.2 & 174.4 & 29.3 & 3.1 & 36.9 & 160.4 \\
\hline 14.6 & 0.3 & 71.2 & 153.0 & 29.4 & 1.7 & -27.8 & 18.5 \\
\hline 14.8 & 2.3 & 14.0 & 167.1 & 29.5 & 2.8 & -47.5 & 29.2 \\
\hline 14.9 & 2.1 & -49.6 & 48.5 & 29.5 & 2.4 & -45.1 & 18.4 \\
\hline 15.2 & 2.2 & 15.2 & 167.5 & 30.0 & 2.2 & 19.0 & 28.2 \\
\hline 15.2 & 0.4 & -42.0 & 18.0 & 30.0 & 0.9 & -41.9 & 15.5 \\
\hline 15.3 & 2.0 & 52.7 & 154.9 & 30.0 & 2.0 & -21.3 & 22.2 \\
\hline 15.5 & 0.6 & 16.3 & 19.0 & 30.8 & 3.0 & -55.1 & 37.2 \\
\hline 15.7 & 1.9 & 1.5 & 172.4 & 31.0 & 1.3 & 70.5 & 128.3 \\
\hline 16.1 & 1.9 & 1.1 & 171.9 & 31.4 & 2.0 & 3.2 & 18.7 \\
\hline 16.3 & 0.6 & 5.3 & 18.6 & 31.9 & 3.1 & -47.1 & 45.3 \\
\hline 16.5 & 1.8 & 68.6 & 134.8 & 32.0 & 4.3 & -0.4 & 162.3 \\
\hline 16.7 & 2.4 & 50.5 & 156.7 & 32.4 & 2.3 & 71.1 & 126.4 \\
\hline 16.8 & 1.4 & -42.0 & 25.8 & 32.3 & 2.8 & 15.0 & 22.0 \\
\hline 17.0 & 2.0 & -44.8 & 32.6 & 32.3 & 2.0 & 69.7 & 158.4 \\
\hline 17.3 & 1.1 & 4.5 & 25.2 & 32.6 & 2.0 & -3.5 & 20.5 \\
\hline 17.4 & 0.9 & -52.8 & 22.7 & 33.0 & 3.6 & 37.4 & 160.4 \\
\hline 17.4 & 1.8 & 20.4 & 177.2 & 33.3 & 2.6 & 20.1 & 28.7 \\
\hline 17.5 & 0.8 & 9.0 & 19.7 & 33.3 & 2.7 & 3.9 & 33.0 \\
\hline 17.6 & 1.0 & -54.3 & 23.5 & 33.4 & 2.3 & 5.1 & 30.8 \\
\hline 18.1 & 1.1 & -0.6 & 17.4 & 33.6 & 1.0 & -45.9 & 27.6 \\
\hline 18.2 & 1.9 & 6.3 & 21.7 & 33.7 & 4.5 & 15.1 & 164.4 \\
\hline 18.3 & 1.6 & 11.2 & 181.2 & 34.0 & 2.2 & -25.9 & 30.0 \\
\hline 18.7 & 2.2 & -21.3 & 30.9 & 34.2 & 0.8 & -25.6 & 22.3 \\
\hline 18.7 & 2.2 & -49.5 & 50.0 & 34.3 & 2.4 & 40.9 & 175.4 \\
\hline 19.3 & 1.9 & 58.6 & 154.2 & 34.6 & 1.8 & 63.8 & 154.0 \\
\hline 19.3 & 1.7 & 2.9 & 19.0 & 34.7 & 3.4 & -54.1 & 45.2 \\
\hline 19.8 & 0.9 & -52.0 & 27.6 & 34.7 & 1.3 & -24.6 & 19.0 \\
\hline 20.2 & 1.8 & -13.8 & 162.0 & 35.2 & 0.7 & 4.7 & 20.4 \\
\hline 20.2 & 1.1 & 73.3 & 147.5 & 35.2 & 2.3 & -2.8 & 19.7 \\
\hline 20.6 & 1.3 & 3.6 & 32.2 & 35.3 & 1.1 & 74.8 & 145.6 \\
\hline 20.7 & 1.9 & 17.6 & 178.6 & 35.7 & 1.7 & 71.5 & 173.3 \\
\hline 21.4 & 1.1 & -9.0 & 19.5 & 35.7 & 2.2 & 77.9 & 151.0 \\
\hline 21.6 & 2.2 & 27.4 & 162.1 & 35.7 & 1.3 & 27.2 & 16.3 \\
\hline 21.7 & 1.7 & -8.2 & 17.9 & 35.8 & 5.2 & 20.8 & 152.1 \\
\hline 21.8 & 2.1 & 72.3 & 103.6 & 36.1 & 1.4 & -37.4 & 37.6 \\
\hline 22.1 & 1.8 & -32.3 & 22.3 & 36.5 & 1.4 & 1.8 & 18.1 \\
\hline 22.2 & 2.1 & 0.3 & 169.6 & 36.7 & 2.3 & 59.8 & 159.4 \\
\hline 22.2 & 1.9 & 56.7 & 175.5 & 37.3 & 2.5 & -47.0 & 37.4 \\
\hline 22.6 & 1.6 & 57.0 & 178.8 & 37.9 & 2.9 & 28.9 & 173.6 \\
\hline 22.6 & 2.8 & -8.3 & 29.3 & 38.5 & 3.2 & 47.4 & 178.8 \\
\hline 22.6 & 3.4 & 8.3 & 160.3 & 38.6 & 2.8 & -11.8 & 25.4 \\
\hline 22.9 & 1.0 & -25.3 & 28.3 & 39.3 & 0.9 & -13.5 & 21.6 \\
\hline 23.2 & 0.7 & 73.3 & 141.5 & 39.6 & 3.8 & -36.3 & 39.7 \\
\hline 23.5 & 1.7 & 14.0 & 18.2 & 39.6 & 1.8 & 9.9 & 18.4 \\
\hline 24.1 & 1.7 & -2.2 & 28.3 & 40.0 & 1.9 & 9.6 & 17.3 \\
\hline 24.4 & 1.4 & 0.5 & 165.9 & 40.1 & 1.5 & -30.7 & 19.4 \\
\hline 24.7 & 3.2 & -1.2 & 30.1 & 40.1 & 2.3 & -1.4 & 181.4 \\
\hline 24.7 & 1.8 & -12.3 & 172.5 & 40.1 & 1.5 & -26.2 & 27.0 \\
\hline 24.8 & 2.7 & 48.4 & 149.3 & 40.1 & 1.8 & 39.8 & 178.8 \\
\hline 24.9 & 2.0 & 14.1 & 25.8 & 40.5 & 1.3 & 71.2 & 177.1 \\
\hline 24.9 & 2.1 & -5.0 & 174.8 & 40.8 & 2.3 & 41.8 & 177.1 \\
\hline 25.0 & 3.0 & -5.1 & 31.1 & 40.8 & 2.2 & 74.1 & 149.4 \\
\hline 25.3 & 3.1 & 7.4 & 167.0 & 41.3 & 2.5 & 10.6 & 182.7 \\
\hline 25.6 & 2.6 & 36.3 & 145.1 & 41.3 & 1.5 & 25.0 & 16.2 \\
\hline 25.8 & 2.2 & -8.4 & 162.8 & 42.1 & 1.4 & -16.8 & 28.5 \\
\hline 25.8 & 2.7 & $\begin{array}{l}0.4 \\
15.0\end{array}$ & 20.5 & 42.7 & 3.4 & -4.5 & 175.3 \\
\hline 26.0 & 0.8 & 73.2 & 157.0 & 42.7 & 1.6 & 3.6 & 19.9 \\
\hline 26.0 & 3.7 & -11.3 & 165.4 & 43.0 & 4.0 & -46.9 & 47.8 \\
\hline 26.3 & 1.6 & -3.5 & 20.2 & 43.4 & 3.2 & -2.3 & 28.6 \\
\hline 26.4 & 1.4 & 69.2 & 152.4 & 43.4 & 1.1 & -51.0 & 25.0 \\
\hline 26.7 & 1.1 & -40.3 & 28.2 & 44.8 & 3.5 & 1.7 & 32.3 \\
\hline 26.7 & 1.0 & -24.0 & 24.5 & 44.9 & 1.8 & -57.6 & 35.3 \\
\hline 27.3 & 1.3 & -74.9 & 37.6 & 45.0 & 1.4 & 31.5 & 12.6 \\
\hline 27.6 & 3.3 & -9.1 & 164.4 & 45.5 & 2.0 & -63.6 & 30.0 \\
\hline 27.7 & 1.8 & -0.1 & 18.8 & 45.7 & 2.1 & 2.5 & 18.8 \\
\hline 27.8 & 1.0 & 73.6 & 155.0 & 46.0 & 1.6 & 73.2 & 144.5 \\
\hline 28.3 & 1.8 & -15.9 & 19.6 & 46.2 & 3.2 & -10.6 & 27.1 \\
\hline 28.4 & 3.8 & 39.1 & 153.6 & 46.7 & 0.9 & -49.9 & 21.9 \\
\hline 28.5 & 0.9 & 79.8 & 156.7 & 47.9 & 1.6 & -13.6 & 22.2 \\
\hline 28.6 & 3.5 & -11.6 & 23.6 & 47.9 & 1.6 & -10.6 & 18.7 \\
\hline 29.1 & 0.9 & 6.5 & 22.1 & 48.2 & 1.3 & -19.9 & 39.2 \\
\hline
\end{tabular}


TABLE $5 b . \quad$ (continued)

\begin{tabular}{|c|c|c|c|}
\hline $\begin{array}{l}\text { Diameter, } \\
\mathbf{k m}\end{array}$ & $\begin{array}{c}\text { Depth, } \\
\text { km }\end{array}$ & $\begin{array}{c}\text { Latitude, } \\
\text { deg }\end{array}$ & $\begin{array}{c}\text { Longitude } \\
\text { deg }\end{array}$ \\
\hline 48.2 & 2.7 & -8.1 & 163.0 \\
\hline 48.3 & 1.9 & -38.9 & 35.3 \\
\hline 48.5 & 2.1 & -54.7 & 27.2 \\
\hline 49.0 & 3.5 & -4.8 & 32.0 \\
\hline 49.1 & 1.2 & -1.2 & 12.8 \\
\hline 49.4 & 1.5 & -26.8 & 28.2 \\
\hline 49.4 & 2.3 & -39.4 & 36.7 \\
\hline 49.8 & 1.7 & 7.0 & 19.1 \\
\hline 49.8 & 2.5 & 8.7 & 181.1 \\
\hline 51.1 & 4.8 & 16.6 & 161.4 \\
\hline 51.5 & 3.1 & -65.2 & -26.8 \\
\hline 51.6 & 2.1 & 80.2 & 160.3 \\
\hline 51.8 & 2.9 & -73.1 & 31.4 \\
\hline 51.8 & 1.9 & -8.2 & 19.3 \\
\hline 53.0 & 1.1 & -15.3 & 21.6 \\
\hline 54.1 & 2.5 & 23.4 & 27.6 \\
\hline 57.1 & 1.8 & 33.6 & 13.3 \\
\hline 57.4 & 3.4 & -4.8 & 17.9 \\
\hline 57.8 & 1.0 & 6.2 & 13.1 \\
\hline 61.5 & 2.6 & 3.6 & 21.9 \\
\hline 62.3 & 1.3 & 0.6 & 18.3 \\
\hline 63.6 & 2.2 & -78.1 & 57.4 \\
\hline 64.6 & 2.2 & -37.8 & 19.7 \\
\hline 65.1 & 1.8 & 25.0 & 19.4 \\
\hline 65.5 & 1.9 & -45.3 & 31.6 \\
\hline 65.7 & 1.9 & -10.4 & 17.9 \\
\hline 66.0 & 1.7 & -1.4 & 19.0 \\
\hline 73.0 & 2.0 & -27.1 & 20.0 \\
\hline 74.9 & 5.9 & -27.9 & 37.5 \\
\hline 75.1 & 3.4 & 20.7 & 25.9 \\
\hline 76.4 & 1.5 & 72.1 & 173.0 \\
\hline 78.8 & 0.6 & 5.0 & 15.6 \\
\hline 79.1 & 1.9 & 20.5 & 23.4 \\
\hline 80.0 & 1.1 & 69.5 & 175.8 \\
\hline 80.2 & 4.8 & -14.5 & 28.5 \\
\hline 82.4 & 2.8 & -42.9 & 30.0 \\
\hline 83.3 & 1.1 & 27.0 & 13.0 \\
\hline 83.3 & 3.1 & 23.2 & 26.7 \\
\hline 84.9 & 2.6 & 0.7 & 16.6 \\
\hline 85.2 & 3.0 & 13.3 & 25.6 \\
\hline 85.8 & 2.4 & 82.2 & 129.3 \\
\hline 86.8 & 2.8 & -14.8 & 19.4 \\
\hline 88.5 & 1.8 & -41.1 & 18.6 \\
\hline 95.2 & 4.9 & -33.2 & 35.5 \\
\hline 97.5 & 1.7 & -27.4 & 34.8 \\
\hline 100.3 & 3.9 & -50.9 & 26.7 \\
\hline 105.8 & 1.2 & 37.4 & 12.6 \\
\hline 113.8 & 2.8 & -45.3 & 21.5 \\
\hline 124.5 & 2.1 & -26.8 & 31.9 \\
\hline 129.9 & 2.0 & -22.6 & 14.9 \\
\hline 170.8 & 0.5 & 72.1 & 154.3 \\
\hline 173.5 & 3.3 & -48.0 & 21.0 \\
\hline
\end{tabular}

the spectrometer's ground trace were not detected, those data may represent a selected subset of craters on Mars (i.e., the deepest). In addition, albedo variations have not been successfully modeled and appear to influence topography derived from the ultraviolet spectrometer. Hence in the size range 5-50 $\mathrm{km}$, available depth/diameter data are inconclusive.

Finally, at crater diameters ranging from 20 to $400 \mathrm{~km}$, earth-based radar range measurements provide estimates of crater depths for 31 craters located within a strip included by latitudes $18^{\circ} \mathrm{S}$ through $13^{\circ} \mathrm{S}$ and by longitudes $107^{\circ} \mathrm{W}$ through $358^{\circ} \mathrm{W}$ (R. Terrile, unpublished data, 1973). The accuracy of the depth measurements varies from about 60 to $150 \mathrm{~m}$ and averages about $100 \mathrm{~m}$. The dimensions of the radar resolution cell (spot size) were approximately $8 \mathrm{~km} \mathrm{E-W}$ and $80 \mathrm{~km} \mathrm{N-S}$, suggesting that depths measured for smaller craters may be underestimates.

\section{ObSERVATIONS}

Qualitative characteristics of depth/diameter curves such as those presented in Figures 2-7 have been discussed by numerous authors [e.g., Baldwin, 1963; Pike, 1974; Gault et al., 1975]. Craters appear to exist in two distinct depth/diameter populations characterized by power laws. The change from the one population to the other occurs over rather narrow depth and diameter ranges and is characterized by a distinct change in the slope of the depth/diameter curve. The junction of the two populations is commonly called the 'break in slope.' Note in Figures 2-6, especially in Figures 4 (bottom left) and 5 (top left), that a distinct fraction of the fresh craters lie above the average population of craters at diameters above the break in slope. In the size range $10-30 \mathrm{~km}$ these deeper craters preserve the depth/diameter relationship characteristic of smaller craters below the change in slope. A detailed discussion of the general nature and interpretation of this and other depth/diameter relationships is presented by Malin and Dzurisin [1976].

\section{Observation of Terrain-Correlated Degradation}

Lunar and mercurian crater populations indicate the existence of terrain-correlated degradational phenomena. Specific evidence will be discussed for each planet separately.

A difference in the depth/diameter relationship exists on Mercury between craters on smooth plains and craters on intercrater plains and in heavily cratered terrain (Figure 3). There is virtually no shallowing of visually degraded smooth plains craters, even though similarly sized craters on inter-

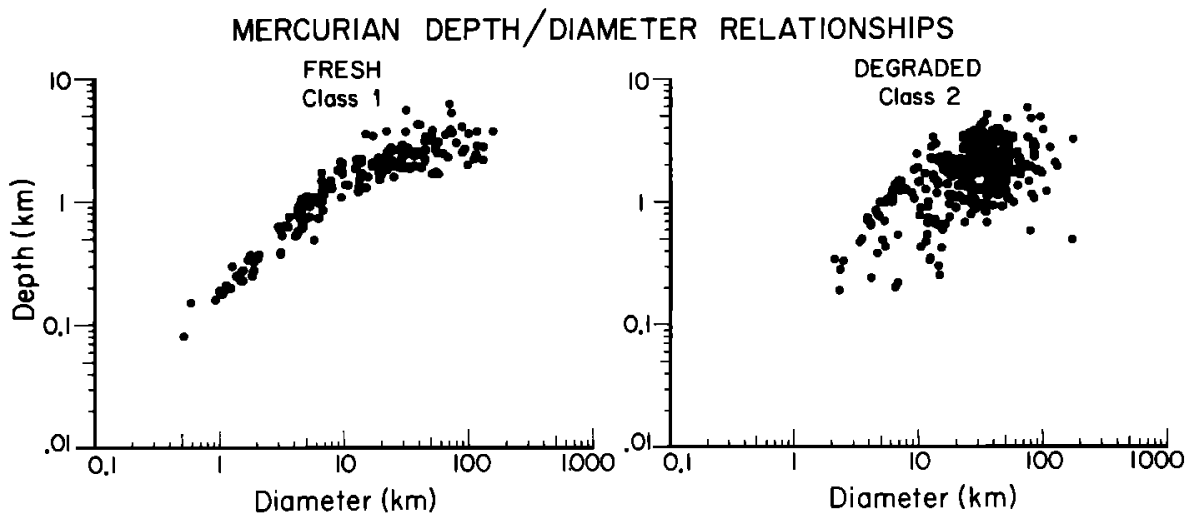

Fig. 2. Depth/diameter curves for fresh and degraded craters on Mercury. 


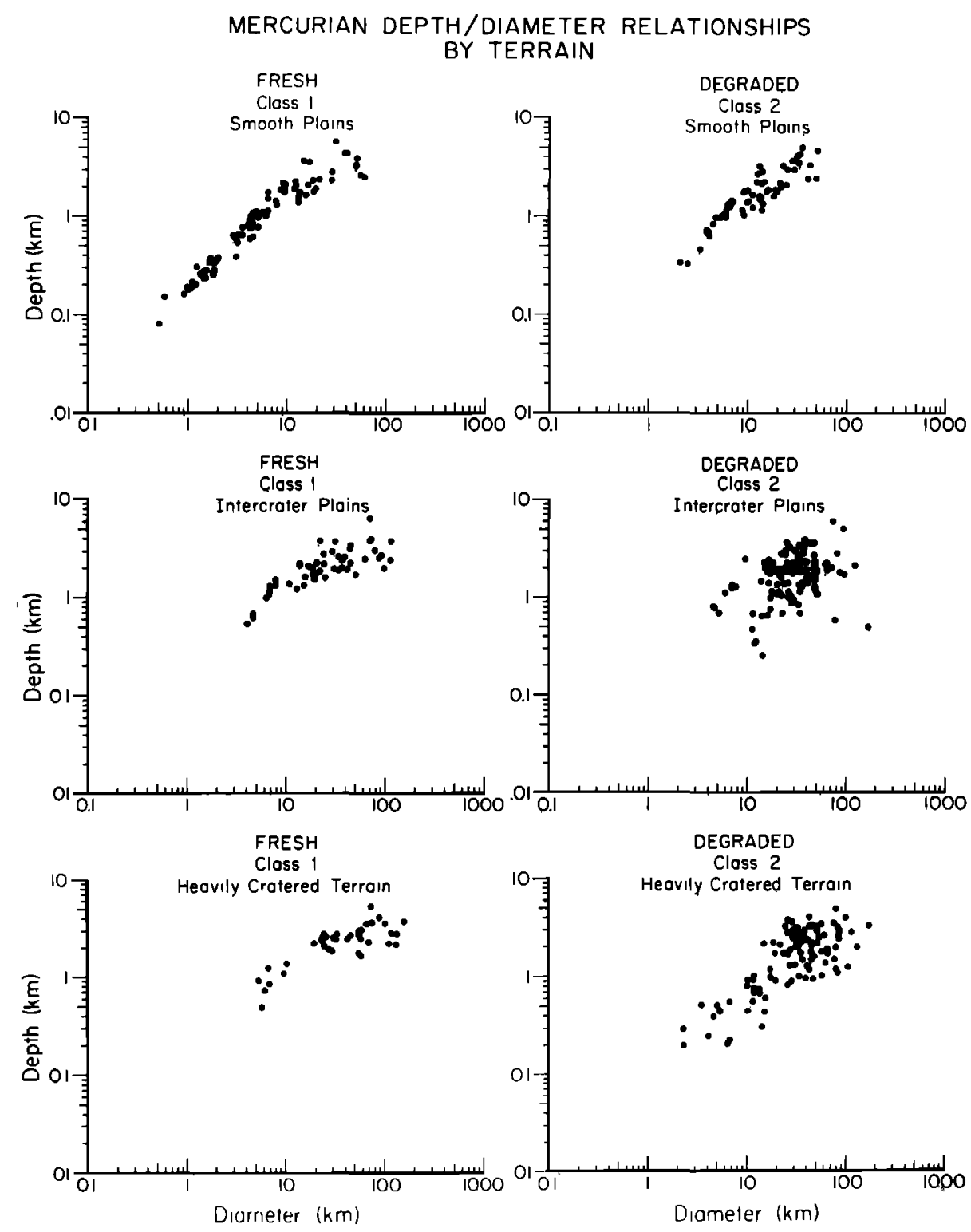

Fig. 3. Depth/diameter relationships for mercurian craters on smooth plains, intercrater plains, and within heavily cratered terrain.

crater plains or within heavily cratered terrain display great variations in depth. The break in slope for mercurian craters occurs at about $9.8-\mathrm{km}$ diameter and $1.6-\mathrm{km}$ depth, as determined by least squares analysis (Table 7 ). There are no apparent depth/diameter differences between crater populations on the intercrater plains and within the heavily cratered terrain.

On the moon, similar characteristics are observed. The degraded crater population within the terrae is similar to that on the mercurian intercrater plains and within the heavily cratered terrain, displaying a large range in depth. A few visually degraded lunar mare craters show depth variations, but these can be attributed to the action of locally restricted processes (e.g., mare flooding). Specifically, the degraded curve for mare craters of classes 2-5 shows two distinct populations with different slopes. The upper curve mimics in all respects the fresh curve and is composed entirely of class 2 craters in Arthur's scheme. The lower curve for craters of classes 3-5 consists of craters either flooded by the mare-forming process or inundated by debris from large impacts of local proximity.
These depth-degraded craters of classes 3-5 form a statistically small population on the maria. There are proportionally fewer visually degraded craters on lunar maria than on mercurian smooth pląins (Tables 1 and 3). For lunar craters the break in slope occurs at roughly $8.7-\mathrm{km}$ diameter and $1.6-\mathrm{km}$ depth from Arthur's data and at 10.7-km diameter and 2.1-km depth from Pike's data (as determined by least squares analyses, Table 7).

\section{SPECulations}

Depth/diameter relations for Mercury and the moon display remarkably similar forms, suggesting similar degrees of landform degradation. Impact erosion, as described quantitatively for the moon by Soderblom [1970] and Soderblom and Lebofsky [1972], may be responsible for the proportionally greater number of visually degraded craters on the mercurian smooth plains, owing to the greater areal density of small craters relative to the lunar maria. However, although significant visual degradation occurs at all crater diameters, no 

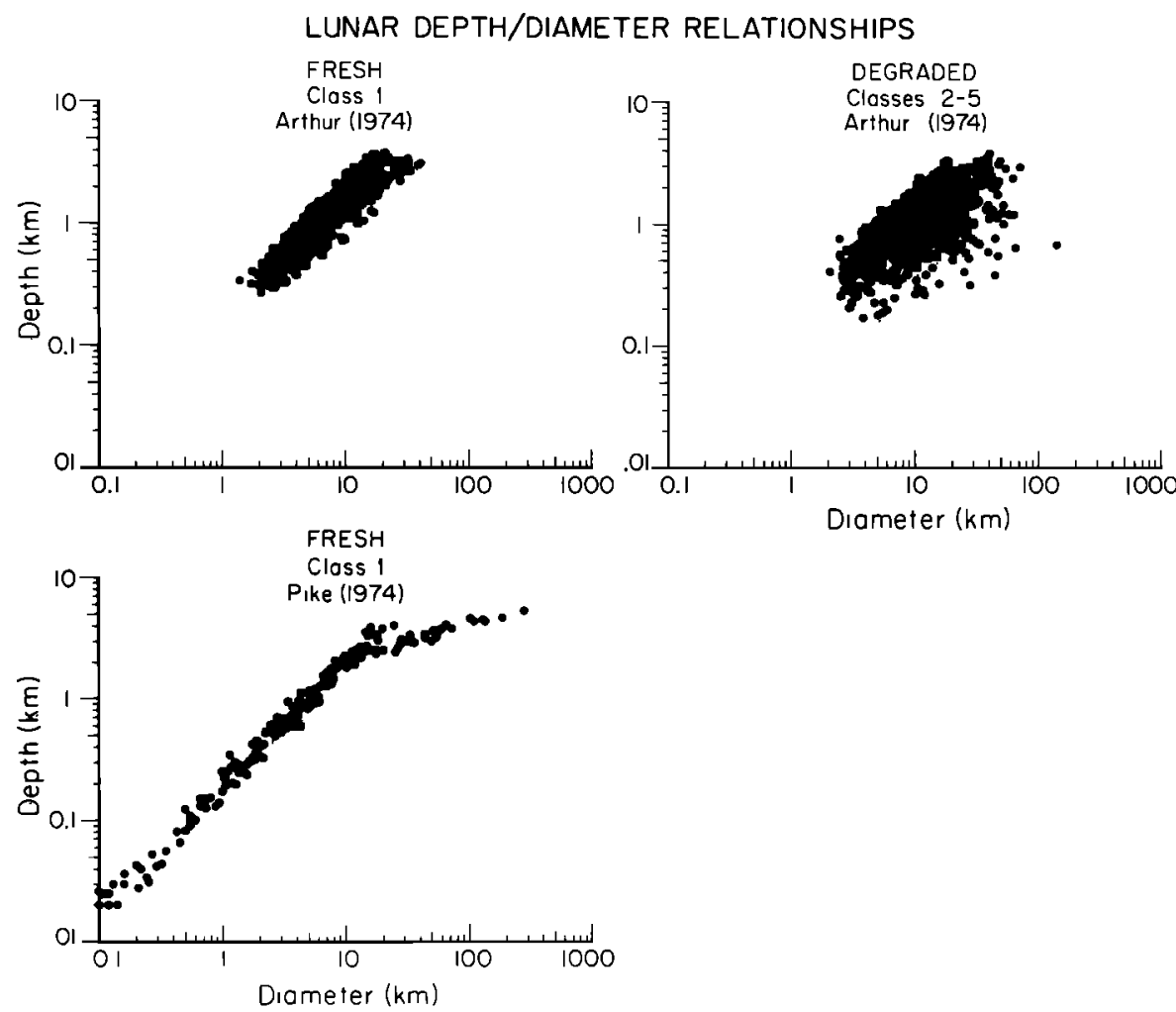

Fig. 4. Depth/diameter relationships for fresh and degraded lunar craters from Lunar Orbiter 4 and Apollo metric camera photography.

mercurian smooth plains craters display significant shallowing. This suggests that the amount of material mobilized since the formation of smooth plains has been insufficient to significantly shallow even the smallest craters observed. This in turn is not fonsistent with the Soderblom/Lebofsky impact erosion model, in which significant visual degradation results from the reduction of wall slopes and the accompanying crater shallowing. Thus while impact erosion remains a plausible hypothesis, available information suggests the action of other mechanisms which involve the transport of relatively small volumes of surficial materials (e.g., thermally induced creep).

In the preceding sections, Mars was not discussed. The family of martian craters (Figure 7) shows few of the characteristic trends of the depth/diameter relationships displayed on Mercury and the moon. The martian depth/diameter curve shows no clearly defined break in slope but does exhibit considerable depth variation. In general, martian craters are shallower than their lunar or mercurian counterparts.

It is important to investigate the relative effectiveness of transport mechanisms to determine the primary and secondary participants in martian surface modification. From the available evidence it seems most likely that deposition, and not erosion, is the primary mode for martian degradation [ $\mathrm{Wil}$ helms, 1974]. Evidence for the moon suggests that deposition by ballistic transport is responsible for much of the crater degradation seen there [Head, 1975]. However, several investigations [Soderblom et al., 1974; Chapman, 1974; Arvidson, 1974] suggest that the martian atmosphere is largely responsible for transport of material associated with landform deterioration. Even the present tenuous atmosphere is capable of considerable transport [Ward et al., 1974], and transient higher-pressure episodes cannot be ruled out.

Major depth degradation on both Mercury and the moon occurs only in the more heavily cratered terrain and the intercrater plains. This suggests that the degradational process, whatever its form, was active before formation of the oldest smooth plains surfaces on both planets. It seems reasonable to associate the degradation with impact-related phenomena, which, at least for the moon, occurred early in its history. Similar degrees of degradation suggest that Mercury and Mars may record a similar history, although the time scales and absolute fluxes remain in question. It thus appears that preplains degradation followed by postplains relative quiescence may be a universal trait of each of the early histories of the terrestrial planets.

Impact cratering is the currently favored process believed responsible for landform degradation. Head [1975] proposes pelting of lunar craters by impacting bodies and, more importantly, lateral transport of material ballistically, either by primary impacts or by secondary impacts associated with large craters and basins. Oberbeck [1975] describes in some detail the ballistic processes associated with the ejection of material from lunar craters and the erosive capability of this ejecta. $\mathrm{N}$. J. Trask (unpublished data, 1976), Trask and Guest [1975], and Murray et al. [1975] invoke similar phenomena to explain mercurian crater degradation, ascribing the greater relative deterioration of landforms to the areal concentration of secondary ejecta resulting from the larger gravitational acceleration. Cintala et al. [1975] similarly apply this hypothesis to Mars.

These processes, however, may not play as important a role on either Mars or Mercury as was suggested by the work of these authors. The greater areal density of mercurian secondary craters may be offset by the restriction in radial distribution which accompanies this areal density increase [Gault et al., 1975] and by the lower density of large primary craters 


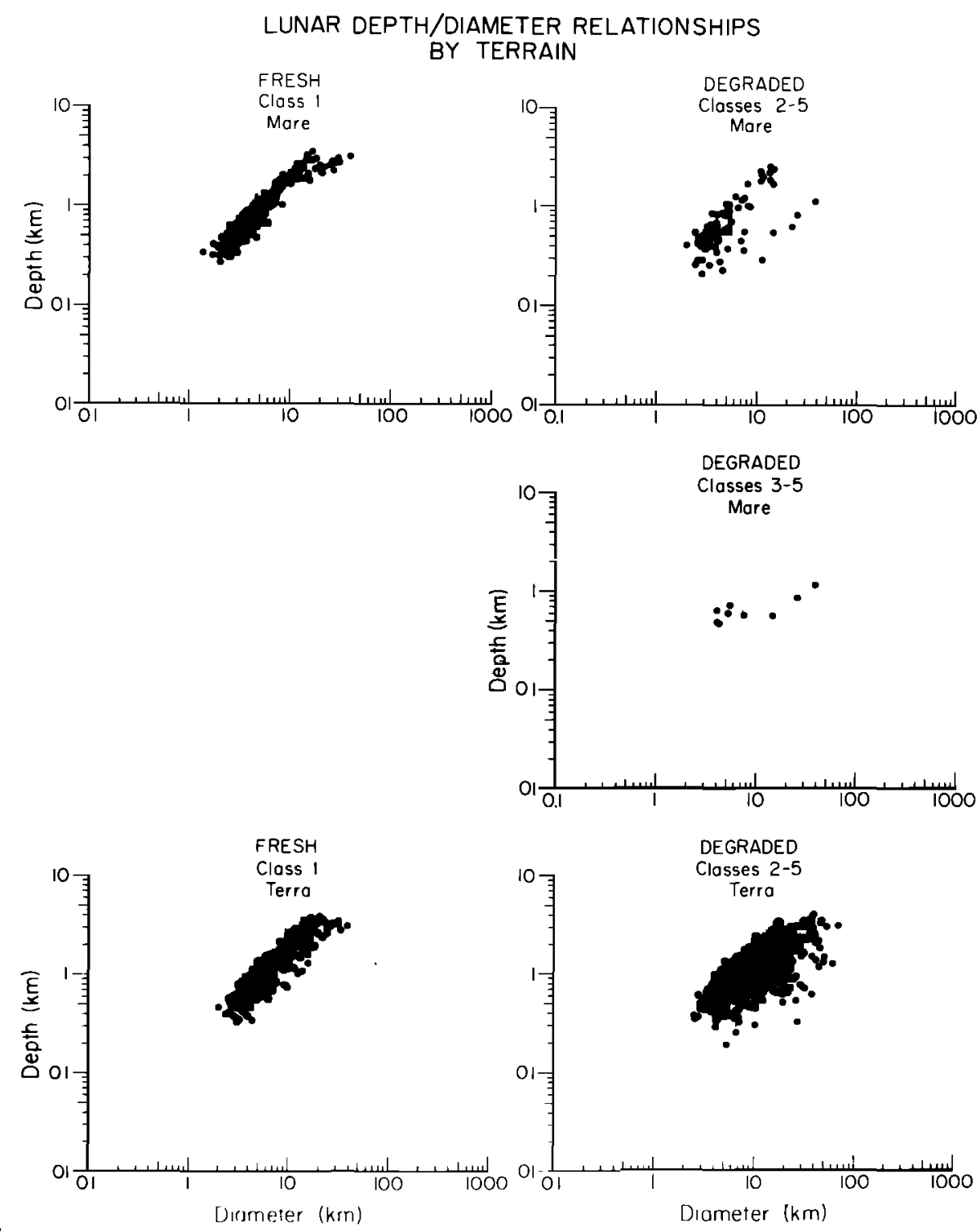

Fig. 5. Depth/diameter information for lunar craters from Arthur [1974] by physiographic province.

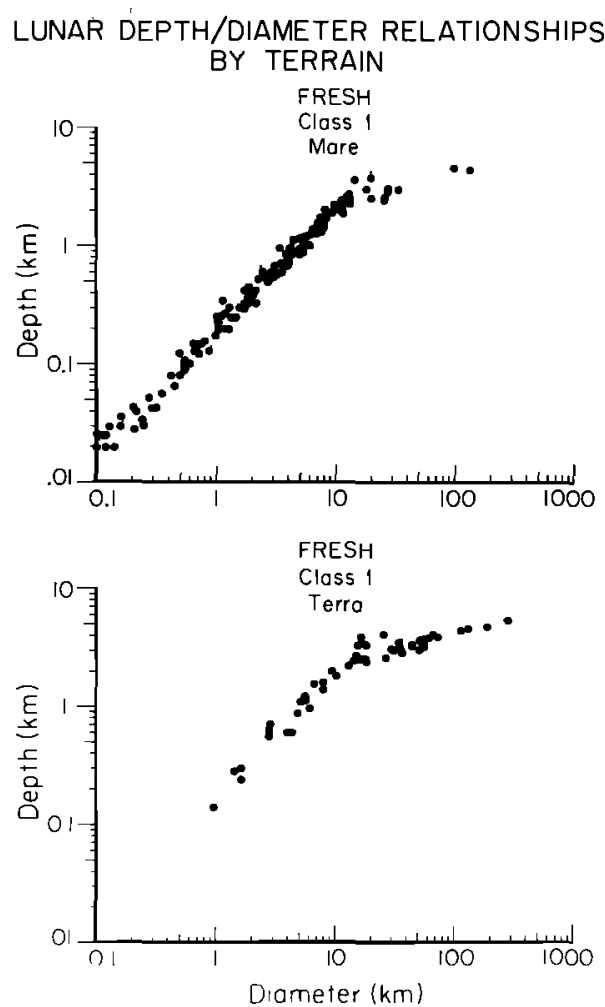

Fig. 6. Depth/diameter curves for fresh lunar mare and terra craters from data of Pike [1974].
[Murray et al., 1974; Malin, 1976b]. Additionally, for Mars the evidence for atmospheric and volcanic modification appears far more convincing than that for impact-related phenomena. In view of these observations, alternative processes operative on the same time scale warrant discussion.

We wish to explore here an alternative, nonballistic hypothesis which is consistent with the depth/diameter data but which is motivated primarily by observations of Mercury and Mars, where the early surface histories appear to have been dominated by formation of large expanses of intercrater plains. Malin [1976a] noted several characteristics of martian intercrater plains (found within the heavily cratered terrain) which bear on their nature and origin. Specifically, he observed that the plains consisted of a stratified sequence of competent and incompetent rock units and that the strata were uniform in thickness over large distances. In some areas he recognized a distinct, resistant cap rock which displayed marelike ridges. His observations indicated that intercrater plains formation ceased abruptly early in martian history. He also noted that erosional processes (e.g., channeling, fretting, and chaos formation) presumably involving volatile materials (i.e., water) operated during the period of intercrater plains formation and ceased shortly thereafter. Malin [1976a] suggested that competent units within intercrater plains are most likely of volcanic origin, and he argued from the uniform thickness over extended distances of less competent units that these were most likely eolian deposits of fine materials (e.g., impact- 
TABLE 6a. Martian Crater Depth/Diameter Data (Near-Terminator Shadow Analysis)

\begin{tabular}{|c|c|c|c|c|c|}
\hline \multicolumn{2}{|c|}{ DAS 8299349* } & \multicolumn{2}{|c|}{ DAS $12499162 \uparrow$} & \multicolumn{2}{|c|}{ DAS $12901203 \ddagger$} \\
\hline $\begin{array}{c}\text { Diameter, } \\
\mathrm{km}\end{array}$ & $\begin{array}{c}\text { Depth, } \\
\text { km }\end{array}$ & $\begin{array}{c}\text { Diameter, } \\
\text { km }\end{array}$ & $\underset{\text { km }}{\text { Depth, }}$ & $\begin{array}{c}\text { Diameter, } \\
\mathbf{k m}\end{array}$ & $\begin{array}{c}\text { Depth, } \\
\text { km }\end{array}$ \\
\hline 0.8 & 0.03 & 0.3 & 0.01 & 0.4 & 0.02 \\
\hline 0.8 & 0.03 & 0.3 & 0.02 & 0.4 & 0.03 \\
\hline 0.8 & 0.04 & 0.3 & 0.02 & 0.5 & 0.03 \\
\hline 0.8 & 0.03 & 0.3 & 0.01 & 0.5 & 0.04 \\
\hline 0.8 & 0.04 & 0.3 & 0.02 & 0.5 & 0.02 \\
\hline 0.8 & 0.04 & 0.3 & 0.02 & 0.5 & 0.03 \\
\hline 0.8 & 0.03 & 0.3 & 0.02 & 0.5 & 0.02 \\
\hline 0.8 & 0.04 & 0.3 & 0.01 & 0.5 & 0.04 \\
\hline 0.8 & 0.03 & 0.3 & 0.01 & 0.5 & 0.03 \\
\hline 0.9 & 0.04 & 0.3 & 0.01 & 0.5 & 0.03 \\
\hline 0.9 & 0.03 & 0.3 & 0.01 & 0.5 & 0.04 \\
\hline 0.9 & 0.05 & 0.4 & 0.03 & 0.5 & 0.03 \\
\hline 1.1 & 0.03 & 0.4 & 0.02 & 0.6 & 0.05 \\
\hline 1.1 & 0.07 & 0.4 & 0.02 & 0.6 & 0.04 \\
\hline 1.1 & 0.07 & 0.4 & 0.02 & 0.6 & 0.06 \\
\hline 1.2 & 0.08 & 0.4 & 0.02 & 0.6 & 0.04 \\
\hline 1.3 & 0.10 & 0.4 & 0.02 & 0.6 & 0.02 \\
\hline 1.4 & 0.09 & 0.4 & 0.02 & 0.6 & 0.05 \\
\hline 1.4 & 0.09 & 0.5 & 0.05 & 0.6 & 0.05 \\
\hline 1.4 & 0.09 & 0.5 & 0.04 & 0.6 & 0.02 \\
\hline 2.2 & 0.14 & 0.5 & 0.03 & 0.6 & 0.04 \\
\hline 2.4 & 0.05 & 0.5 & 0.06 & 0.6 & 0.04 \\
\hline 2.6 & 0.20 & 0.5 & 0.02 & 0.6 & 0.04 \\
\hline \multirow[t]{27}{*}{2.6} & 0.18 & 0.5 & 0.02 & 0.6 & 0.06 \\
\hline & & 0.5 & 0.04 & 0.6 & 0.02 \\
\hline & & 0.5 & 0.02 & 0.6 & 0.03 \\
\hline & & 0.5 & 0.02 & 0.6 & 0.01 \\
\hline & & 0.5 & 0.02 & 0.6 & 0.04 \\
\hline & & 0.5 & 0.03 & 0.6 & 0.03 \\
\hline & & 0.5 & 0.04 & 0.6 & 0.03 \\
\hline & & 0.5 & 0.02 & 0.7 & 0.05 \\
\hline & & 0.6 & 0.06 & 0.7 & 0.06 \\
\hline & & 0.6 & 0.06 & 0.8 & 0.04 \\
\hline & & 0.6 & 0.06 & 1.5 & 0.08 \\
\hline & & 0.6 & 0.04 & 2.2 & 0.05 \\
\hline & & 0.6 & 0.02 & & \\
\hline & & 0.6 & 0.02 & & \\
\hline & & 0.6 & 0.05 & & \\
\hline & & 0.6 & 0.06 & & \\
\hline & & 0.6 & 0.02 & & \\
\hline & & 0.6 & 0.03 & & \\
\hline & & 0.6 & 0.04 & & \\
\hline & & 0.6 & 0.02 & & \\
\hline & & 0.7 & 0.02 & & \\
\hline & & 0.8 & 0.06 & & \\
\hline & & 0.8 & 0.07 & & \\
\hline & & 0.8 & 0.07 & & \\
\hline & & 1.3 & 0.07 & & \\
\hline & & 1.4 & 0.13 & & \\
\hline & & 1.6 & 0.08 & & \\
\hline
\end{tabular}

*Latitude, $40.4^{\circ}-42.0^{\circ}$; longitude, $108.6^{\circ}-111.1^{\circ}$.

† Latitude, $44.4^{\circ}-45.4^{\circ}$; longitude, $159.7^{\circ}-161.2^{\circ}$.

‡Latitude, $21.6^{\circ}-22.5^{\circ}$; longitude, $253.5^{\circ}-254.6^{\circ}$.

generated debris, weathering prồducts, volcanic ash). He invoked a major, early atmosphere to explain both the great amounts of uniformily deposited debris and the widespread distribution of erosional landforms whose geneses apparently involved volatiles. The early cessation of intercrater plains formation and the accompanying decline of volatile-related erosion led Malin to speculate that the evolution of the martian atmosphere was in some way linked to the early bombardment of the planet.

Murray et al. [1975] urge a volcanic origin for the formation of intercrater plains on Mercury, since potential alternative mechanisms (e.g., impact erosion and deposition, accretionary melting, atmospheric erosion) are judged to be inconsistent with areal restriction of impact ejecta and the survival of 'saturated' cratered surfaces. Malin [1976c], on the basis of observations of transectional relationships between large craters and intercrater plains, suggests that the formation of the intercrater plains occurred throughout the period of heavy bombardment. The existence of similar ancient plains on the moon may indicate that it also went through a phase of intercrater plains formation.

We suggest that all three planets record nonballistic processes which acted prior to the formation of the oldest smooth plains and, by analogy with lunar chronology, may have been correlated at least temporally with early bombardment. Whether the processes reflect the composition of the impacting 
TABLE 6b. Martian Crater Depth/Diameter Data [Leighton. 1966]

\begin{tabular}{|c|c|c|c|}
\hline $\begin{array}{c}\text { Diameter, } \\
\text { km }\end{array}$ & $\begin{array}{l}\text { Depth, } \\
\text { km }\end{array}$ & $\begin{array}{c}\text { Diameter, } \\
\mathrm{km}\end{array}$ & $\begin{array}{c}\text { Depth, } \\
\text { km }\end{array}$ \\
\hline 6.0 & 0.1 & 10.9 & 0.2 \\
\hline 6.0 & 0.1 & 10.9 & 0.2 \\
\hline 6.0 & 0.2 & 10.9 & 0.5 \\
\hline 6.4 & 0.2 & 15.1 & 0.3 \\
\hline 6.4 & 0.2 & 17.1 & 0.4 \\
\hline 7.1 & 0.2 & 17.1 & 0.4 \\
\hline 7.1 & 0.2 & 17.1 & 1.0 \\
\hline 7.1 & 0.1 & 18.1 & 0.5 \\
\hline 8.0 & 0.2 & 22.2 & 0.4 \\
\hline 8.0 & 0.2 & 22.0 & 0.3 \\
\hline 8.0 & 0.2 & 23.1 & 0.2 \\
\hline 9.0 & 0.2 & 24.1 & 0.1 \\
\hline 9.0 & 0.3 & 24.1 & 0.2 \\
\hline 9.0 & 0,4 & 24.1 & 0.4 \\
\hline 10.0 & 0.1 & 28.3 & 0.5 \\
\hline 10.0 & 0.2 & 29.1 & 0.6 \\
\hline 10.0 & 0.2 & 29.8 & 0.7 \\
\hline 10.0 & 0.3 & 30.0 & 0.4 \\
\hline 10.0 & 0.4 & 31.2 & 0.4 \\
\hline \multirow[t]{2}{*}{10.0} & 0.5 & 34.2 & 0.1 \\
\hline & & 36.2 & 0.4 \\
\hline
\end{tabular}

Data are from R. Terrile (unpublished radar data, 1973).

TABLE 6c. Martian Crater Depth/Diameter Data

\begin{tabular}{|c|c|c|c|}
\hline $\begin{array}{c}\text { Diameter, } \\
\text { km }\end{array}$ & $\begin{array}{c}\text { Depth, } \\
\text { km }\end{array}$ & $\begin{array}{l}\text { Latitude, } \\
\text { deg }\end{array}$ & $\begin{array}{c}\text { Longitude, } \\
\text { deg }\end{array}$ \\
\hline 24 & 0.6 & -14.5 & 172 \\
\hline 24 & 0.8 & -14 & 116 \\
\hline 43 & 0.7 & -15 & 272 \\
\hline 48 & 0.9 & -16.5 & 112 \\
\hline 48 & 1.5 & -15 & 287 \\
\hline 48 & 1.5 & -18.5 & 230 \\
\hline 53 & 0.6 & -16 & 358.5 \\
\hline 53 & 0.7 & -15.5 & 262.5 \\
\hline$\$ 3$ & 0.8 & -15 & 335 \\
\hline 53 & 0.9 & -13 & 343 \\
\hline 58 & 0.4 & -14.5 & 116.5 \\
\hline 58 & 0.7 & -18 & 348.5 \\
\hline 58 & 1.0 & -14 & 142.5 \\
\hline 58 & 1.0 & -17 & 299.5 \\
\hline 58 & 1.1 & -15 & 215 \\
\hline 60 & 0.6 & -16 & 107 \\
\hline 63 & 0.6 & -14 & 246 \\
\hline 72 & 1.0 & -14 & 217.5 \\
\hline 77 & 0.9 & -14.5 & 284 \\
\hline 82 & 1.4 & -15 & 262 \\
\hline 87 & 0.8 & -14 & 167 \\
\hline 92 & 1.3 & -14.5 & 331 \\
\hline 97 & 1.0 & -14 & 316.5 \\
\hline 97 & 1.4 & -17 & 211 \\
\hline 101 & 1.6 & -15 & 197.5 \\
\hline 106 & 1.7 & -14 & 156 \\
\hline 116 & 0.7 & -16 & 165 \\
\hline 150 & 2.0 & -14.5 & 185 \\
\hline 237 & 1.2 & -17 & 341 \\
\hline 309 & 1.2 & -15.5 & 229 \\
\hline 449 & 1.5 & -14 & 214 \\
\hline 459 & 1.2 & -15 & 129 \\
\hline
\end{tabular}

Data are from $R$. Terrile (unpublished radar data, 1973).

TABLE 7. Least Squares Fits to Depth/Diameter Data

\begin{tabular}{|c|c|c|c|}
\hline Planet & $\begin{array}{c}\text { Small Craters } \\
\left(\text { Diameter }<D_{b}\right)\end{array}$ & $\begin{array}{c}\text { Large Craters } \\
\text { (Diameter }>D_{b} \text { ) }\end{array}$ & Break in Slope \\
\hline Mercury & $d=0.176 D^{089}$ & $d=0.91 D^{0.28}$ & $\begin{aligned} d_{b} & =1.6 \mathrm{~km} \\
D_{b} & =9.8 \mathrm{~km}\end{aligned}$ \\
\hline $\begin{array}{l}\text { Moon } \\
\quad[\text { Pike, 1974] }\end{array}$ & $d=0.195 D^{1.01}$ & $d=1.05 D^{0.30}$ & $\begin{aligned} d_{b} & =2.1 \mathrm{~km} \\
D_{b} & =10.7 \mathrm{~km}\end{aligned}$ \\
\hline $\begin{array}{l}\text { Moon } \\
\text { [Arthur, 1974] }\end{array}$ & $d=0.182 D^{101}$ & $d=0.61 D^{0.45}$ & $\begin{aligned} d_{b} & =1.6 \mathrm{~km} \\
D_{b} & =8.7 \mathrm{~km}\end{aligned}$ \\
\hline
\end{tabular}

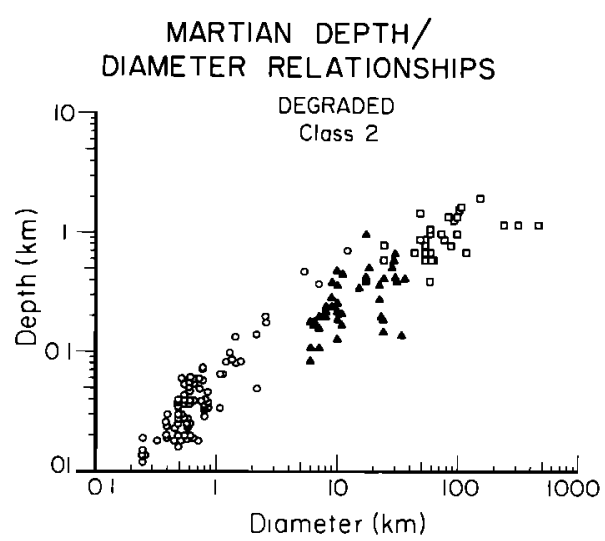

Fig. 7. Depth/diameter relationship for craters on Mars. Circles represent shadow measurements of near-terminator, narrow-angle Mariner 9 images, triangles are from photoclinometric analysis of Mariner 4 images by Leighton [1966], and squares represent earthbased radar range measurements ( $R$. Terrile, unpublished data, 1973). Ultraviolet spectrometer data were unavailable but are suggested to lie somewhat above those of Leighton.

objects (e.g., volatile or radioisotope content), the mode and rate of deposition of heat related to the kinetic energy of the impacting objects, or other phenomena associated with the bombardment cannot be determined at this time. It likewise cannot be established whether these processes were planet specific or common to all terrestrial planets.

Regardless of whether the ballistic or nonballistic hypothesis is applied, it seems likely that the surfaces of at least three terrestrial planets underwent significant modification very early in their histories. This suggests that they record processes which may have acted on the earth, the products of which, however, have since been obscured by other continuing processes.

Acknowledgments. The authors express their appreciation to Richard Terrile of Caltech, who unselfishly provided unpublished analyses of radar observations of martian craters. D.D. was supported by an NSF graduate research fellowship. This research was supported in part by NASA grant NSG7155 and presents the results of one phase of research carried out at the Jet Propulsion Laboratory, California Institute of Technology, sponsored by NASA under contract NAS7-100. Contribution 2648 of the Division of Geological and Planetary Sciences, California Institute of Technology, Pasadena, California.

\section{REFERENCES}

Arthur, D. W. G., (Ed.), The system of lunar craters, quadrant 1 , Contrib. 3, Lunar and Planet. Lab., Univ. of Ariz., Tucson, 1963. Arthur, D. W. G., Lunar crater depths from Orbiter 4 long-focus photographs, Icarus, 23, 116-133, 1974.

Arvidson, R. E., Morphologic classification of martian craters and some implications, Icarus, 22, 264-271, 1974.

Baldwin, R. B., The Measure of the Moon, pp. 128-152, University of Chicago Press, Chicago, Ill., 1963.

Chapman, C. R., Cratering on Mars, 1, Cratering and obliteration history, Icarus, 22, 272-291, 1974.

Cintala, M. J., J. W. Head, and T. A. Mutch, Depth/diameter relationships for martian and lunar craters (abstract), Eos Trans. $A G U$, 56(6), 389, 1975.

Gault, D. E., J. E. Guest, J. B. Murray, D. Dzurisin, and M. C. Malin, Some comparisons of impact craters on Mercury and the moon, $J$. Geophys. Res., 80(17), 2444-2460, 1975.

Head, J. W., Processes of lunar crater degradation: Changes in style with geologic time, Moon, 12, 299-330, 1975.

Leighton, R. B., The photographs from Mariner 4, Sci. Amer., 214(4), 54-68, 1966.

Malin, M. C., Studies of the surface morphology of the planet Mars, Ph.D. dissertation, Calif. Inst. of Technol., Pasadena, $1976 a$.

Malin, M. C., Comparison of large crater and multiringed basin populations on Mars, Mercury, and the moon, Proc. Lunar Sci. Conf. $7 \mathrm{th}$, in press, $1976 \mathrm{~b}$. 
Malin, M. C., Observations of intercrater plains on Mercury, Geophys. Res. Lett., 3, in press, $1976 c$.

Malin, M. C., and D. Dzurisin, Modification of fresh crater landforms: Evidence from Mercury and the moon, in Proceedings of the Conference on Comparisons of Mercury and the Moon, Lunar Science Institute, Houston, Tex., 1976.

Murray, B. C., M. J. S. Belton, G. E. Danielson, M. E. Davies, D. E. Gault, B. Hapke, B. O'Leary, R. G. Strom, V. Suomi, and N. J. Trask, Mercury's surface: Preliminary description and interpretation from Mariner 10 pictures, Science, 185, 169-179, 1974.

Murray, B. C., R. G. Strom, N. J. Trask, and D. E. Gault, Surface history of Mercury: Implications for terrestrial planets, J. Geophys. Res., 80(17), 2508-2514, 1975.

Oberbeck, V. R., The role of ballistic erosion and sedimentation in lunar stratigraphy, Rev. Geophys. Space Phys., 13, 337-362, 1975.

Pike, R. J., Depth/diameter relations of fresh lunar craters: Revision from spacecraft data, Geophys. Res. Lett., I(7), 291-294, 1974.

Pohn, H. A., and T. W. Offield, Lunar crater morphology and relative age determination of lunar geologic units, 1, Classification, U.S. Geol. Surv. Prof Pap., 700-C, 153-162, 1970.
Soderblom, L. A., A model for small-impact erosion applied to the lunar surface, J. Geophys. Res., 75, 2655-2661, 1970.

Soderblom, L. A., and L. A. Lebofsky, Technique for rapid determination of relative ages of lunar areas from orbital photography, $J$. Geophys. Res., 77, 279-296, 1972.

Soderblom, L. A., C. D. Condit, R. A. West, B. M. Herman, and T. J. Kreidler, Martian planetwide crater distribution: Implications for geologic history and surface processes, Icarus, 22, 239-263, 1974.

Trask, N. J., and J. E. Guest, Preliminary geologic terrain map of Mercury, J. Geophys. Res., 80(17), 2461-2477, 1975.

Ward, W. R., B. C. Murray, and M. C. Malin, Climatic variations on Mars, 2, Evolution of carbon dioxide atmosphere and polar caps, $J$. Geophys. Res., 79, 3387-3395, 1974.

Wilhelms, D. E., Comparison of martian and lunar geologic provinces, J. Geophys. Res., 79, 3933-3941, 1974.

(Received November 14, 1975; revised August 5, 1976; accepted September 3, 1976.) 